\title{
Parental Perspectives on Immunizations: Impact of the COVID-19 Pandemic on Childhood Vaccine Hesitancy
}

\author{
Kaidi He ${ }^{1}\left[\right.$ 'Wendy J. Mack ${ }^{2} \cdot$ Michael Neely $^{1} \cdot$ Laura Lewis $^{3} \cdot$ Vikram Anand $^{1}$ \\ Accepted: 14 July 2021 / Published online: 23 July 2021 \\ (c) The Author(s), under exclusive licence to Springer Science+Business Media, LLC, part of Springer Nature 2021
}

\begin{abstract}
Childhood vaccine hesitancy has been studied extensively before the COVID-19 pandemic. The pandemic presented new barriers to pediatric vaccinations. Furthermore, the development of COVID-19 vaccines has complicated factors underlying vaccine hesitancy. We performed a cross-sectional mobile phone-based survey at Children's Hospital Los Angeles querying parents regarding perspectives on vaccines before and during the pandemic. Our primary aim was to understand the impact of the pandemic on routine childhood vaccine hesitancy. Secondarily, we examined intent to vaccinate, COVID-19 vaccine hesitancy, and key contributing demographic factors. Among 252 participants, we found overall increased childhood vaccine hesitancy $(p=0.006)$, increased risk perception $(p=0.006)$, and unchanged vaccine confidence during the COVID-19 pandemic. Increased hesitancy did not translate into decreased intent to vaccinate with routine childhood vaccines or influenza vaccines. During the pandemic, households with higher income (50-99 K, > $100 \mathrm{~K}$ ) correlated with decreased routine childhood vaccine hesitancy, while Hispanic ethnicity and African American race had increased risk perception. For COVID-19 vaccine hesitancy, households with higher income (>100 K) correlated with decreased hesitancy, while non-White ethnicity and race had increased risk perception. We found that routine childhood vaccine hesitancy increased during the COVID-19 pandemic, mainly due to increased risk perception. Key contributing demographic factors behind both childhood vaccine hesitancy and COVID-19 vaccine hesitancy included household income and race. Understanding factors behind routine childhood vaccine hesitancy is crucial to maintaining pediatric vaccination rates and promoting vaccine confidence during and after the COVID-19 pandemic.
\end{abstract}

Keywords COVID-19 pandemic $\cdot$ Childhood vaccine hesitancy $\cdot$ COVID-19 vaccines

\section{Background}

The COVID-19 pandemic has altered the landscape of pediatric vaccines. Social distancing and stay-at home orders presented new barriers to vaccine administration. The Centers for Disease Control and Prevention (CDC) reported a significant decline in pediatric vaccines one week following

Vikram Anand

vanand@chla.usc.edu

1 Present Address: Department of Infectious Diseases, Children's Hospital Los Angeles, 4650 Sunset Blvd MS \#51, Los Angeles, CA 90027, USA

2 Department of Preventive Medicine, Keck School of Medicine, University of Southern California, Los Angeles, CA, USA

3 Department of Pediatrics, Keck School of Medicine, University of Southern California, Los Angeles, CA, USA the national declaration of emergency due to the pandemic [1]. The Michigan Care Improvement Registry reported that up-to-date vaccines declined to $<50 \%$ among children less than or equal to 2 years of age [2]. At the same time, pharmaceutical companies partnered with CDC to develop safe COVID-19 vaccines. It is unclear how parental perspectives towards routine childhood vaccines and COVID-19 vaccines have been affected by the pandemic; these perspectives may affect future widespread implementation of COVID-19 vaccines among children.

Vaccine hesitancy has been defined by the World Health Organization (WHO) as "refusal of vaccines despite availability of vaccine services" [3]. More recently, it has been defined by Kempe et al. as "a motivational state of being conflicted about or opposed to getting vaccinated" [4]. Global data shows that vaccine hesitancy is prominent in more than $90 \%$ of countries worldwide [5]. Vaccine hesitancy can apply to all routine childhood vaccines or specific 
vaccines, with highest hesitancy directed towards influenza and HPV vaccines (26.1 and $25.6 \%$ of parents respectively) [6]. The recent development of COVID-19 vaccines has further pushed vaccine hesitancy to the forefront of discussion.

Childhood vaccine hesitancy has been extensively studied; however, currently, there are no existing studies on the impact of the pandemic on routine childhood vaccine hesitancy. Studies published during the pandemic have reflected data collected in years prior. In July 2020, Kempe et al. showed the rate of parental vaccine hesitancy to be $6.7 \%$ for routine childhood vaccines and over $25 \%$ for influenza vaccines [4]. In December 2020, Santibanez et al. reported that $20 \%$ of parents are hesitant about routine childhood vaccines [7]. Both studies were done in US parents. Notably, they used data from 2018 to 2019, before the pandemic. The majority of vaccine hesitancy literature during the pandemic has been devoted to hesitancy in adults about COVID-19 vaccination for adults. In our review, we found only two publications studying parental COVID-19 vaccine hesitancy for their children: Yigit et al. reported that $56.8 \%$ would vaccinate their child with a domestic COVID-19 vaccine [8] and Rhodes et al. noted parental unwillingness to vaccinate their child with the COVID-19 vaccine [9]. We found only one study on the influence of the pandemic on influenza vaccine hesitancy: Goldman et al. showed that higher level of caregiver concern about having COVID-19 was associated with increased intent to vaccinate against influenza [10].

During the pandemic, substantial misinformation about vaccines has been spread, and there have been concerns that vaccine hesitancy has increased. Simultaneously, there has been significant emphasis on importance of vaccines by community health experts. The interplay of these factors is important to understand for the implementation of pediatric COVID-19 vaccines.

The primary objective of our study was to determine whether the pandemic impacted parental perspectives on routine childhood vaccines in our patient population. Secondary objectives included assessing intent to vaccinate, COVID-19 vaccine hesitancy, and key contributing demographic factors to vaccine hesitancy during the pandemic.

\section{Methods}

\section{Study Population}

We conducted a cross-sectional survey at Children's Hospital Los Angeles (CHLA) and Altamed Pediatric Clinic between September 2020 and February 2021. Parents of patients from General Pediatrics, subspecialty clinics, and inpatient general medical/surgical units were recruited by convenience sampling to complete a mobile phone-based survey about their attitudes towards routine childhood vaccines, influenza vaccines, and COVID-19 vaccines. A parent of any child age 18 years or younger was eligible. Parents of intensive care units and emergency departments were excluded, as the acuity of illness of these patients was expected to interfere with parental participation in the survey. Parents of oncology patients were excluded, as their eligibility for COVID-19 vaccines was unknown at the time of the study. Parents were recruited in clinic waiting rooms and inpatient hospital rooms under the CHLA and Altamed Institutional Review Board -approved "exempt" protocol. Parents were provided with a QR Code to be scanned into their mobile phone with a link to the online survey. Informed consent was established by participation in the survey.

\section{Survey Design}

\section{Focus Groups}

Parents of children up to 18 years of age were recruited through existing University of Southern California (USC) community outreach groups for three focus groups, held from July 2020 to August 2020, to discuss reasons for routine childhood vaccine hesitancy and safety concerns about COVID-19 vaccines. Groups of 5-10 participants were led by a study team physician in English and Spanish in unrecorded Zoom sessions. Sample survey questions were reviewed by participants, poorly worded questions were discarded, and survey questions were rewritten based on the discussions. Participants were awarded a $\$ 20$ gift card upon completion of session.

\section{Survey}

We used questions based on a modified Vaccine Hesitancy Scale (VHS), developed by WHO's Strategic Advisory Group of Experts (SAGE), to assess parents how they felt about routine childhood vaccines and influenza vaccines before and during the pandemic. In addition, we surveyed perspectives on the COVID-19 vaccine. VHS is a 10-item scale where parents answer questions on vaccines with a Likert Scale of "Strongly Agree" to "Strongly Disagree" [11]. VHS item scores are summed to a total VHS score. Items are averaged in two categories, Lack of Confidence and Risk (Table 3), allowing assessment of these two constructs underlying vaccine hesitancy. VHS was developed after extensive global pilot data and literature review. Initially validated with Canadian and French parents of 9-16 year-old children, VHS has since been used in the United States, with a modified version to assess influenza vaccine hesitancy for patients 6 months to 18 years [12]. We used the 9-item scale for childhood immunizations, as prior literature showed that one of the original 10 items was unreliable [13]. We added Item 2 to assess the importance of influenza vaccines. The 
Table 1 Summary of parent demographics $(\mathrm{N}=252)$

\begin{tabular}{|c|c|}
\hline Demographics & Total parents n $(\%)$ \\
\hline \multicolumn{2}{|l|}{ Location } \\
\hline Outpatient (general pediatrics) & $203(80.6 \%)$ \\
\hline Inpatient (general pediatrics) & $24(9.5 \%)$ \\
\hline Outpatient (subspecialty) & $22(8.7 \%)$ \\
\hline No response & $3(1.2 \%)$ \\
\hline \multicolumn{2}{|l|}{ Gender } \\
\hline Female & $210(83.3 \%)$ \\
\hline Male & $42(16.7 \%)$ \\
\hline No response & $0(0 \%)$ \\
\hline \multicolumn{2}{|l|}{ Age (years) } \\
\hline $18-29$ & $66(26.2 \%)$ \\
\hline $30-44$ & $139(55.2 \%)$ \\
\hline $45-54$ & $39(15.5 \%)$ \\
\hline $55-65$ & $6(2.4 \%)$ \\
\hline $65+$ & $1(0 \%)$ \\
\hline No response & $1(0 \%)$ \\
\hline \multicolumn{2}{|l|}{ Political identification } \\
\hline Democrat & $101(40.1 \%)$ \\
\hline Republican & $13(5.2 \%)$ \\
\hline Independent & $11(4.4 \%)$ \\
\hline Other/None & $64(25.4 \%)$ \\
\hline Decline to State & $50(19.8 \%)$ \\
\hline No response & $13(5.2 \%)$ \\
\hline \multicolumn{2}{|l|}{ Highest level of education } \\
\hline Did not graduate from high school & $23(9.1 \%)$ \\
\hline High school & $123(48.8 \%)$ \\
\hline Bachelor's degree & $68(27.0 \%)$ \\
\hline Master's degree & $13(5.2 \%)$ \\
\hline Post-graduate degree & $11(4.4 \%)$ \\
\hline No response & $14(5.6 \%)$ \\
\hline \multicolumn{2}{|l|}{ Household income } \\
\hline$<49 \mathrm{~K}$ & $132(52.4 \%)$ \\
\hline $50 \mathrm{~K}$ to $99 \mathrm{~K}$ & $48(19.0 \%)$ \\
\hline$>100 \mathrm{~K}$ & $21(8.3 \%)$ \\
\hline Don't know/decline to state & $37(14.7 \%)$ \\
\hline No response & $14(5.6 \%)$ \\
\hline \multicolumn{2}{|l|}{ Race/ethnicity } \\
\hline White & $27(10.7 \%)$ \\
\hline Hispanic & $156(61.9 \%)$ \\
\hline African American & $14(5.6 \%)$ \\
\hline Other/multiple & $36(14.3 \%)$ \\
\hline No response & $19(7.5 \%)$ \\
\hline
\end{tabular}

original VHS used a 5-point Likert scale. We excluded the "Neutral" option, as prior studies have shown this to be effective in decreasing social desirability bias [4]. Numeric values were assigned 5= "Strongly Disagree," $4=$ "Somewhat Disagree," $2=$ "Somewhat Agree," and 1= "Strongly Agree." Higher item scores indicated higher hesitancy, and items were reverse-coded when applicable. Higher scores in Lack of Confidence and Risk indicated decreased confidence and increased risk perception, respectively.

A separate set of questions based on VHS was generated about COVID-19 vaccine hesitancy. Items assessing efficacy and benefit of COVID-19 vaccines were omitted because COVID-19 vaccines were not yet available at the time of survey administration. Item 2 was also omitted, as it pertained to influenza vaccines. Both childhood vaccine and COVID-19 vaccine Lack of Confidence and Risk were related to demographic variables: location, gender, age, political identification, highest level of education, household income, and race/ethnicity.

We assessed whether experiencing the COVID-19 pandemic was associated with a change in intent to vaccinate with routine childhood vaccines and influenza vaccines. We asked parents to recall how many vaccines their child received before the pandemic and state how many routine vaccines they planned to receive during the pandemic ("None," "Most," "Almost All," or "All"). We asked parents to recall if their child had received an influenza vaccine before the pandemic. We asked if they planned to or had already received vaccination against influenza for 2020-2021 season during the pandemic ("Yes" and "No").

Survey questions underwent review by a panel of physicians from CHLA Infectious Diseases and General Pediatrics, and USC Preventive Medicine. They were translated from English to Spanish and programmed into Qualtrics. All completed survey data were anonymous without linkage to individual participant identifiers.

\section{Statistical Analyses}

\section{Childhood Vaccine Hesitancy Before and During the Pandemic}

Paired t-tests were used to compare VHS total scores, Lack of Confidence and Risk scores before and during the pandemic. Mean (SD) values, paired mean differences and 
Table 2 Vaccine hesitancy scale (VHS) items used to assess childhood vaccine hesitancy and COVID-19 vaccine hesitancy

\begin{tabular}{|c|c|c|}
\hline VHS item & Childhood vaccine hesitancy & COVID-19 vaccine hesitancy \\
\hline & Before/During COVID-19: & \\
\hline 1 & $\begin{array}{l}\text { I feel/felt that childhood vaccines are important for my child's } \\
\text { health }\end{array}$ & A future COVID-19 vaccine is important for my child's health \\
\hline 2 & $\begin{array}{l}\text { I feel/felt that the influenza vaccine is important for my child's } \\
\text { health }^{ \pm}\end{array}$ & Omitted \\
\hline 3 & $\begin{array}{l}\text { I feel/felt that getting vaccines is a good way to protect my child } \\
\text { from disease }\end{array}$ & $\begin{array}{l}\text { A future COVID-19 vaccine will be a good way to protect my } \\
\text { child from disease }\end{array}$ \\
\hline 4 & I feel/felt that childhood vaccines are effective & Omitted \\
\hline 5 & $\begin{array}{l}\text { I feel/felt that having my child vaccinated is important for the } \\
\text { health of others in my community }\end{array}$ & $\begin{array}{l}\text { Having my child vaccinated with a future COVID-19 vaccine is } \\
\text { important for the health of others in my community }\end{array}$ \\
\hline 6 & $\begin{array}{l}\text { I feel/felt that all childhood vaccines offered by the government } \\
\text { program in my community are beneficial }\end{array}$ & Omitted \\
\hline 7 & $\begin{array}{l}\text { I feel/felt that the information I receive about childhood vaccines } \\
\text { from my child's healthcare provider is reliable and trustworthy }\end{array}$ & $\begin{array}{l}\text { The information I receive about a future COVID-19 vaccine from } \\
\text { my child's healthcare provider is reliable and trustworthy }\end{array}$ \\
\hline 8 & $\begin{array}{l}\text { I did/do what my doctor or healthcare provider recommends } \\
\text { about vaccines for my child }\end{array}$ & $\begin{array}{l}\text { I will do what my doctor or healthcare provider recommends } \\
\text { about a future COVID-19 vaccine }\end{array}$ \\
\hline 9 & I feel/felt that new vaccines carry more risks than older vaccines & $\begin{array}{l}\text { A future COVID-19 vaccine carries more risks than older vac- } \\
\text { cines }\end{array}$ \\
\hline 10 & I was/am concerned about serious adverse effects of vaccines & $\begin{array}{l}\text { I am concerned about serious adverse effects of a future COVID- } \\
19 \text { vaccine }\end{array}$ \\
\hline
\end{tabular}

${ }^{ \pm}$Item 2 was not part of original VHS (added to assess influenza vaccine hesitancy)

Table 3 Summary of vaccine hesitancy scale (VHS) scores for childhood vaccine hesitancy before and during the COVID-19 pandemic

\begin{tabular}{|c|c|c|c|c|c|c|}
\hline \multicolumn{7}{|c|}{ VHS items and summary score: before COVID-19, during COVID-19, and differences } \\
\hline \multirow[t]{2}{*}{ VHS Item } & \multicolumn{2}{|c|}{ Before COVID-19 } & \multicolumn{2}{|c|}{ During COVID-19 } & \multirow[t]{2}{*}{ p-value } & \multirow[t]{2}{*}{$\mathrm{n}$} \\
\hline & Median (IQR) & $\% 4$ or 5 & Median (IQR) & $\% 4$ or 5 & & \\
\hline 1: Vaccines are important & $1(1,2)$ & 7.30 & $1(1,2)$ & 7.30 & 0.74 & 192 \\
\hline 2: Influenza vaccines are important & $1(1,2)$ & 14.97 & $1(1,2)$ & 13.90 & 0.13 & 187 \\
\hline 3: Vaccines protect from disease & $1(1,2)$ & 8.07 & $1(1,2)$ & 7.53 & 0.92 & 186 \\
\hline 4: Vaccines are effective & $1(1,2)$ & 8.75 & $1(1,2)$ & 9.29 & 0.73 & 183 \\
\hline 5: Vaccines are important for community health & $1(1,2)$ & 6.48 & $1(1,2)$ & 6.48 & 0.17 & 185 \\
\hline 6: Vaccine are beneficial & $1(1,2)$ & 8.70 & $1(1,2)$ & 11.42 & 0.048 & 184 \\
\hline 7: Information on vaccines is reliable & $1(1,2)$ & 6.01 & $1(1,2)$ & 8.20 & 0.17 & 183 \\
\hline $\begin{array}{l}\text { 8: I do what my healthcare provider recommends } \\
\text { about vaccines }\end{array}$ & $1(1,2)$ & 5.58 & $1(1,2)$ & 7.26 & 0.035 & 179 \\
\hline 9: New vaccines carry more risks & $4(2,5)$ & 71.75 & $4(4,5)$ & 76.83 & 0.01 & 177 \\
\hline 10: I am concerned about vaccine side effects & $4(2,5)$ & 74.43 & $4(4,5)$ & 80.69 & 0.043 & 176 \\
\hline \multicolumn{7}{|l|}{ VHS category } \\
\hline Total VHS: mean (SD) & $2.00(0.73)$ & & $2.07(0.74)$ & & 0.006 & 171 \\
\hline Lack of confidence: mean (SD) & $1.51(0.88)$ & & $1.56(0.92)$ & & 0.15 & 174 \\
\hline Risk: mean (SD) & $3.70(1.15)$ & & $3.88(1.11)$ & & 0.006 & 176 \\
\hline
\end{tabular}

Lack of Confidence is comprised of average of Items 1, 3-8. Risk is comprised of average of Items 9, 10

Test for differences Before vs. During COVID-19 item scores by Wilcoxon signed rank test

Test for differences Before vs. During COVID-19 category scores (total, lack of confidence, risk) by paired $t$ test

Items with statistically significant difference before and during COVID-19 


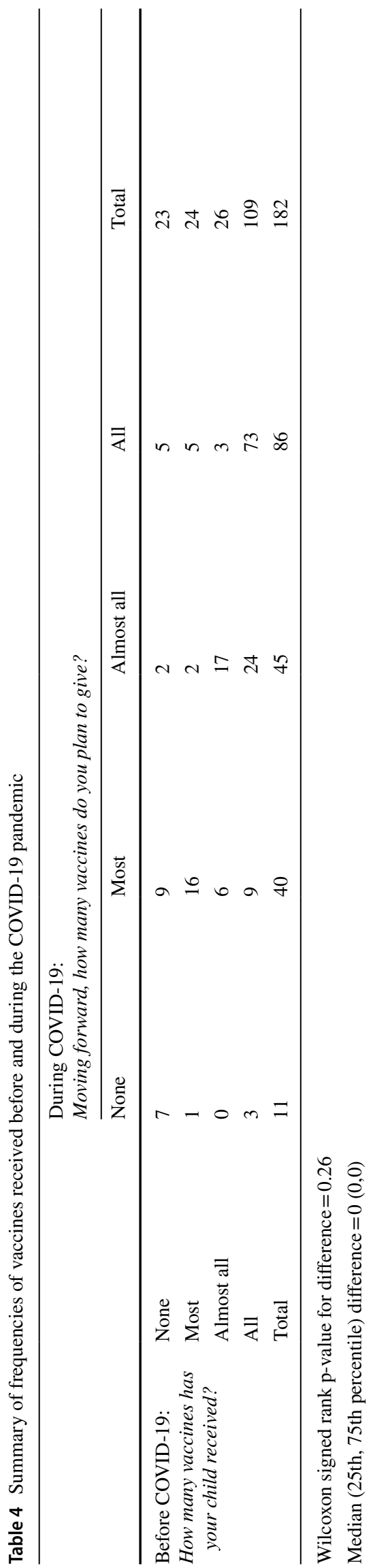

95\% confidence intervals were calculated. For specific item responses, comparisons before and during the COVID-19 pandemic used Wilcoxon signed rank tests, as the data were not normally distributed. Specific item responses were summarized as median, 25th, 75th percentiles, and "\% 4 or 5," the percentage of responses that indicated greater vaccine hesitancy ( $4=$ "Somewhat Disagree" and 5 = "Strongly Disagree").

\section{Associations with Vaccine Hesitancy}

General linear models were used to relate demographic factors to Lack of Confidence and Risk for (1) childhood vaccine hesitancy (2) COVID-19 vaccine hesitancy. Model residuals were evaluated for normality and homoscedasticity. A p-value of $<0.05$ was considered statistically significant.

\section{Prior History of Vaccination vs. Future Intent to Vaccinate}

Tests for matched proportions were used to analyze whether there was a difference in distribution of answers ("None," "Most," "Almost All," "All") for number of vaccines a child received before the pandemic and number planned during the pandemic. Tests for matched proportions were run to analyze if there was a difference in proportion of those who answered "Yes" for having vaccinated for influenza before the pandemic and those who planned to vaccinate during the pandemic.

\section{Results}

A total of 252 surveys was obtained; 175 participants completed $90 \%$ or more of the survey. For incomplete surveys, most unanswered questions came from the end of the survey. All incomplete surveys were included in final data analysis. However, when the participant did not answer questions for both before and during the pandemic, the individual question was excluded. Most respondents were from outpatient clinic $(80.6 \%)$, female $(83.3 \%)$, between 30 and 44 years old $(55.2 \%)$, Democratic $(40.1 \%)$, graduated high school (48.8\%), had annual income <49 K (52.4\%), and were of Hispanic ethnicity (61.9\%) (Table 1 ), reflective of the surrounding community.

\section{Childhood Vaccine Hesitancy}

Table 2 displays VHS item numbers and corresponding questions, and Table 3 summarizes individual VHS items scores and Lack of Confidence and Risk scores before and during the COVID-19 pandemic. Relative to before the pandemic, total VHS scores during the pandemic were higher. Magnitude of change was summarized by the difference 
in means ("During" minus "Before"). For total VHS, the mean difference was 0.075 (95\% confidence interval $(0.021$, $0.128), p=0.006)$. For Lack of Confidence, the mean difference was 0.048 (95\% confidence interval $(-0.018,0.115)$, $\mathrm{p}=0.15)$. For Risk, the mean difference was $0.173(95 \%$ confidence interval $(0.051,0.295), p=0.006)$. For Items $6-10$, " $\% 4$ or 5 " increased during the pandemic, indicating greater vaccine hesitancy. The magnitude of change was $2.72 \%$ for statements on "Vaccines are beneficial," (Item $6, p=0.048), 1.68 \%$ for "I do what my healthcare provider recommends about vaccines" (Item $8, p=0.035$ ), $5.08 \%$ for "New vaccines carry more risks" (Item $9, \mathrm{p}=0.01$ ), and $6.26 \%$ for "I am concerned about vaccine side effects" (Item $10, p=0.043$ ). For all other items, the difference in scores before and during the pandemic was not statistically significant ( $\mathrm{p}>0.05)$.

We found no statistically significant change in frequencies of routine vaccines received before the pandemic and vaccines planned during the pandemic. During the pandemic, $47.3 \%$ ( 86 of 182) planned to receive "All" vaccines and $6.0 \%$ (11 of 182) planned to receive "No" vaccines, which was a $-6.5 \%$ and $-12.6 \%$ decrease respectively, compared to vaccines received before the pandemic. Neither change was statistically significant $(p=0.26)$ (Table 4$)$.

\section{Importance of Influenza Vaccines}

We found no statistically significant change from before to during the pandemic in how parents perceived the importance of influenza vaccines. This is reflected in our added VHS Item 2 ("The influenza vaccine is important for my child's health"). For before and during the pandemic, the data shows no statistically significant difference in scores $(\mathrm{p}=0.13)$ (Table 3). Furthermore, intent to vaccinate against influenza did not significantly change. During the pandemic, $81.5 \%$ (119 of 146) answered "Yes," a change of 2.7\%, which was not statistically significant $(\mathrm{p}=0.37)$ (Table 5).

\section{Demographic Correlates of Vaccine Hesitancy}

Tables 6 and 9 display statistically significant associations of demographic variables with childhood vaccine hesitancy and COVID-19 vaccine hesitancy for Lack of Confidence and Risk, assessed during the pandemic. For childhood vaccine hesitancy, household income $>100 \mathrm{~K}(\mathrm{p}=0.031)$, Hispanic ethnicity $(\mathrm{p}=0.038)$, and Other/Multiple race $(\mathrm{p}=0.039)$ were associated with increased confidence (decreased Lack of Confidence scores) (Table 6). Age 45-54 years $(\mathrm{p}=0.012)$, Master's Degree $(\mathrm{p}=0.039)$, and household income 50-99 K ( $p=0.03)$ were associated with decreased risk perception (decreased Risk score), while Hispanic ( $\mathrm{p}=0.0001)$, African American $(\mathrm{p}=0.0012)$, and Other/ 
Table 6 Associations of demographic variables with childhood vaccine hesitancy, assessed during the COVID-19 pandemic_lack of confidence

\begin{tabular}{|c|c|c|c|}
\hline Independent variable & $\mathrm{n}$ & Beta (SE) & p-value \\
\hline Location & 175 & & \\
\hline Outpatient (general pediatrics) & & Referent & \\
\hline Inpatient (general pediatrics) & & $-0.17(0.21)$ & 0.42 \\
\hline Outpatient (subspecialty) & & $0.16(0.24)$ & 0.52 \\
\hline Gender & 178 & & \\
\hline Male & & $-0.35(0.18)$ & \\
\hline \multicolumn{4}{|l|}{ Female } \\
\hline \multicolumn{4}{|l|}{ Non-binary } \\
\hline Age (years) & 178 & & \\
\hline $18-29$ & & Referent & \\
\hline $30-44$ & & $-0.10(0.17)$ & 0.55 \\
\hline $45-54$ & & $-0.22(0.22)$ & 0.32 \\
\hline $55-65$ & & $-0.62(0.48)$ & 0.20 \\
\hline $65+$ & & $-0.37(0.92)$ & 0.69 \\
\hline Political identification & 178 & & \\
\hline Democrat & & Referent & \\
\hline Republican & & $0.30(0.27)$ & 0.27 \\
\hline Independent & & $-0.39(0.36)$ & 0.28 \\
\hline Other/none & & $-0.25(0.17)$ & 0.15 \\
\hline Decline to state & & $0.05(0.18)$ & 0.79 \\
\hline Highest level of education & 177 & & \\
\hline Did not graduate HS & & $-0.44(0.28)$ & 0.11 \\
\hline HS graduate & & Referent & \\
\hline Bachelor's degree & & $-0.14(0.16)$ & 0.39 \\
\hline Master's degree & & $-0.22(0.29)$ & 0.46 \\
\hline Post-graduate & & $-0.22(0.34)$ & 0.52 \\
\hline Household Income & 177 & & \\
\hline$<49 \mathrm{~K}$ & & Referent & \\
\hline $50-99 \mathrm{~K}$ & & $-0.32(0.18)$ & 0.07 \\
\hline$>100 \mathrm{~K}$ & & $-0.49(0.23)$ & 0.031 \\
\hline Don't know/decline to state & & $-0.23(0.20)$ & 0.25 \\
\hline Racelethnicity & 177 & & \\
\hline White & & Referent & \\
\hline Hispanic & & $-0.41(0.20)$ & 0.038 \\
\hline African American & & $0.22(0.31)$ & 0.48 \\
\hline Other/multiple & & $-0.52(0.25)$ & 0.039 \\
\hline Primary language & 178 & & \\
\hline English & & Referent & \\
\hline Spanish & & $-0.26(0.16)$ & 0.09 \\
\hline Other & & $-0.23(0.31)$ & 0.46 \\
\hline Number of children in household & 174 & $-0.079(0.062)$ & 0.20 \\
\hline Number tested COVID positive & 177 & $0.013(0.055)$ & 0.81 \\
\hline Number believed to have had COVID & 176 & $0.067(0.062)$ & 0.28 \\
\hline Number of recommended vaccines received & 177 & & \\
\hline None & & $0.39(0.20)$ & 0.054 \\
\hline Most & & $0.92(0.20)$ & $<0.0001$ \\
\hline Almost all & & $0.50(0.19)$ & 0.007 \\
\hline All & & Referent & \\
\hline Had flu vaccine & 177 & & \\
\hline Yes & & Referent & \\
\hline No & & $0.58(0.15)$ & 0.0002 \\
\hline Can't remember & & $0.99(0.23)$ & $<0.0001$ \\
\hline
\end{tabular}

Positive beta scores indicate decreased (increased lack of) confidence 
Table 7 Associations of demographic variables with childhood vaccine hesitancy, assessed during the COVID-19 pandemic-risk

\begin{tabular}{|c|c|c|c|}
\hline Independent variable & \# in analysis & Beta (SE) & p-value \\
\hline Location & 176 & & \\
\hline Outpatient (general pediatrics) & & Referent & \\
\hline Inpatient (general pediatrics) & & $0.03(0.27)$ & 0.92 \\
\hline Outpatient (subspecialty) & & $0.32(0.29)$ & 0.28 \\
\hline Gender & 179 & & \\
\hline Male & & $0.02(0.22)$ & 0.93 \\
\hline Female & & Referent & \\
\hline \multicolumn{4}{|l|}{ Non-binary } \\
\hline Age (years) & 178 & & \\
\hline $18-29$ & & Referent & \\
\hline $30-44$ & & $-0.20(0.20)$ & 0.32 \\
\hline $45-54$ & & $-0.68(0.27)$ & 0.012 \\
\hline $55-65$ & & $-0.34(0.58)$ & 0.56 \\
\hline $65+$ & & $0.91(1.13)$ & 0.42 \\
\hline Political identification & 179 & & \\
\hline Democrat & & Referent & \\
\hline Republican & & $-0.59(0.34)$ & 0.08 \\
\hline Independent & & $0.18(0.440$ & 0.68 \\
\hline Other/none & & $0.05(0.21)$ & 0.80 \\
\hline Decline to state & & $0.37(0.22)$ & 0.10 \\
\hline Highest level of education & 177 & & \\
\hline Did not graduate HS & & $-0.10(0.35)$ & 0.77 \\
\hline HS graduate & & Referent & \\
\hline Bachelor's degree & & $-0.09(0.19)$ & 0.65 \\
\hline Master's degree & & $-0.75(0.36)$ & 0.039 \\
\hline Post-graduate & & $0.25(0.42)$ & 0.54 \\
\hline Household income & 177 & & \\
\hline$<49 \mathrm{~K}$ & & Referent & \\
\hline $50-99 \mathrm{~K}$ & & $-0.48(0.22)$ & 0.03 \\
\hline$>100 \mathrm{~K}$ & & $-0.23(0.28)$ & 0.41 \\
\hline Don't know/decline to state & & $-0.06(0.26)$ & 0.82 \\
\hline Racelethnicity & 178 & & \\
\hline White & & Referent & \\
\hline Hispanic & & $0.98(0.25)$ & 0.0001 \\
\hline African American & & $1.27(0.38)$ & 0.0012 \\
\hline Other/multiple & & $0.88(0.31)$ & 0.006 \\
\hline Primary language & 179 & & \\
\hline English & & Referent & \\
\hline Spanish & & $0.11(0.20)$ & 0.59 \\
\hline Other & & $-0.07(0.39)$ & 0.86 \\
\hline Number of children in household & 175 & $0.156(0.077)$ & 0.045 \\
\hline Number tested COVID positive & 178 & $0.112(0.068)$ & 0.10 \\
\hline Number believed to have had COVID & 177 & $0.091(0.077)$ & 0.24 \\
\hline Number of recommended vaccines received & 178 & & \\
\hline None & & $0.42(0.26)$ & 0.11 \\
\hline Most & & $0.31(0.26)$ & 0.23 \\
\hline Almost all & & $-0.19(0.24)$ & 0.44 \\
\hline All & & Referent & \\
\hline Had flu vaccine & 178 & & \\
\hline Yes & & Referent & \\
\hline No & & $0.23(0.20)$ & 0.27 \\
\hline
\end{tabular}


Table 7 (continued)

\begin{tabular}{llll}
\hline Independent variable & \# in analysis & Beta (SE) & p-value \\
\hline Can't remember & $-0.24(0.30)$ & 0.43 \\
\hline
\end{tabular}

Positive beta scores indicate increased perception of risk

Multiple race $(\mathrm{p}=0.006)$ were associated with increased risk perception (Table 7).

For COVID-19 vaccine hesitancy, male sex $(\mathrm{p}=0.013)$, age 45-54 years $(p=0.032)$, less than High School education $(\mathrm{p}=0.014)$, and household income $>100 \mathrm{~K}(\mathrm{p}=0.008)$ were associated with increased confidence (decreased Lack of Confidence score) (Table 8). Declining to state political identification $(p=0.018)$, African American $(p=0.0001)$, Hispanic $(p=0.0003)$, and Other/Multiple race $(p=0.011)$ respondents had increased risk perception (increased Risk score), whereas higher education such as Master's Degree $(p=0.009)$ and Post-graduate Degree $(p=0.025)$ were associated with decreased risk perception (Table 9).

\section{Discussion}

Our study shows that childhood vaccine hesitancy during the COVID-19 pandemic increased by a small but statistically significant degree, with higher total VHS scores during the pandemic compared with before. The greatest change during the pandemic for childhood vaccine hesitancy came from increased risk perception, as evidenced by higher Risk scores. The Risk category is comprised of questions concerning vaccine side effects, suggesting vaccine safety profile is a driving factor behind vaccine hesitancy. Overall, vaccine confidence, as measured by Lack of Confidence category, remained unchanged. Lack of Confidence is assessed through extent of parental agreement with healthcare provider trustworthiness and perceived benefit of vaccines. Our data shows that during the pandemic, there was increased doubt about benefit of vaccines offered by the government and trustworthiness of healthcare provider recommendations. This may reflect growing public concern regarding the extent of government involvement in the COVID-19 vaccine development process [14] and global distrust for scientific research [15], both noted during the pandemic. Previous publications have found an "anti-vaccination infodemic on social media" [16], with increase in anti-vaccine searches during the pandemic [17]. An increase in vaccine misinformation would support our findings of increased vaccine hesitancy. However, as parental perspectives on importance and effectiveness of vaccines were unchanged, most items assessed under Lack of Confidence were unchanged, resulting in unchanged overall confidence.

While overall vaccine hesitancy and risk perception increased, these changes had no statistically significant effect on plan for vaccinating with routine childhood vaccines and influenza vaccines. Parental willingness to vaccinate with "All" and "No" childhood vaccines before and during the pandemic did not change. Large awareness campaigns have been instituted to promote vaccinations; perhaps these influenced vaccine confidence. Our findings on intent to vaccinate against influenza differ from a recent study by Goldman et al., which found a $15.8 \%$ increase in plan to vaccinate against influenza after the pandemic (our increase of $2.7 \%$ was not statistically significant) [10]. Literature prior to the pandemic shows that in parents who are hesitant about influenza vaccines, 34 to $47 \%$ still vaccinated their child [7], which agrees with our findings that intent to vaccinate with influenza vaccine remains largely unchanged. We found no statistically significant difference in how parents viewed the importance of influenza vaccines during compared to before the COVID-19 pandemic. This may be because our survey respondents already felt influenza vaccines were important (the median VHS Score was 1 = "Strongly Agree" before the pandemic).

We identified key demographic correlates for childhood vaccine hesitancy and COVID-19 vaccine hesitancy in terms of confidence and risk perception. For childhood vaccine hesitancy, higher income households had less hesitancy, with both increased confidence $(>100 \mathrm{~K})$ and decreased risk perception (50-99 K). Our findings agree with those of Boyle et al., who found that higher income households have higher vaccine acceptance [18]. For childhood vaccine hesitancy, Hispanic ethnicity had increased confidence, but also increased risk perception. African American race had no association with confidence, however, did have increased risk perception. For COVID-19 vaccine hesitancy, African American race had decreased confidence, and both Hispanic ethnicity and African American race had increased risk perception. Similarly, Khubchandani et al. found that COVID-19 vaccine hesitancy was higher among African Americans and Hispanics compared to Whites [19]. While Khubchandani et al. and others have found Republicans to 


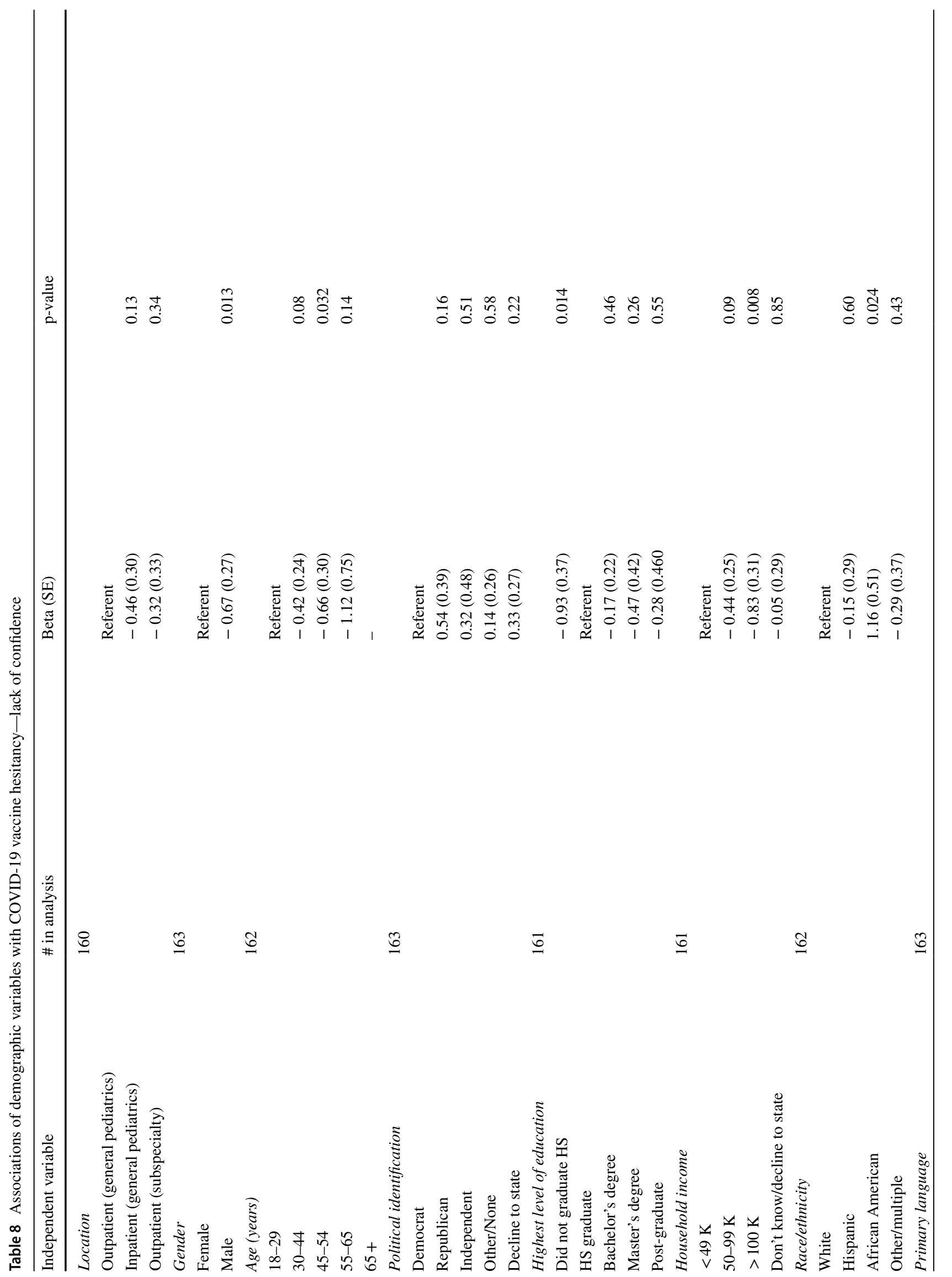




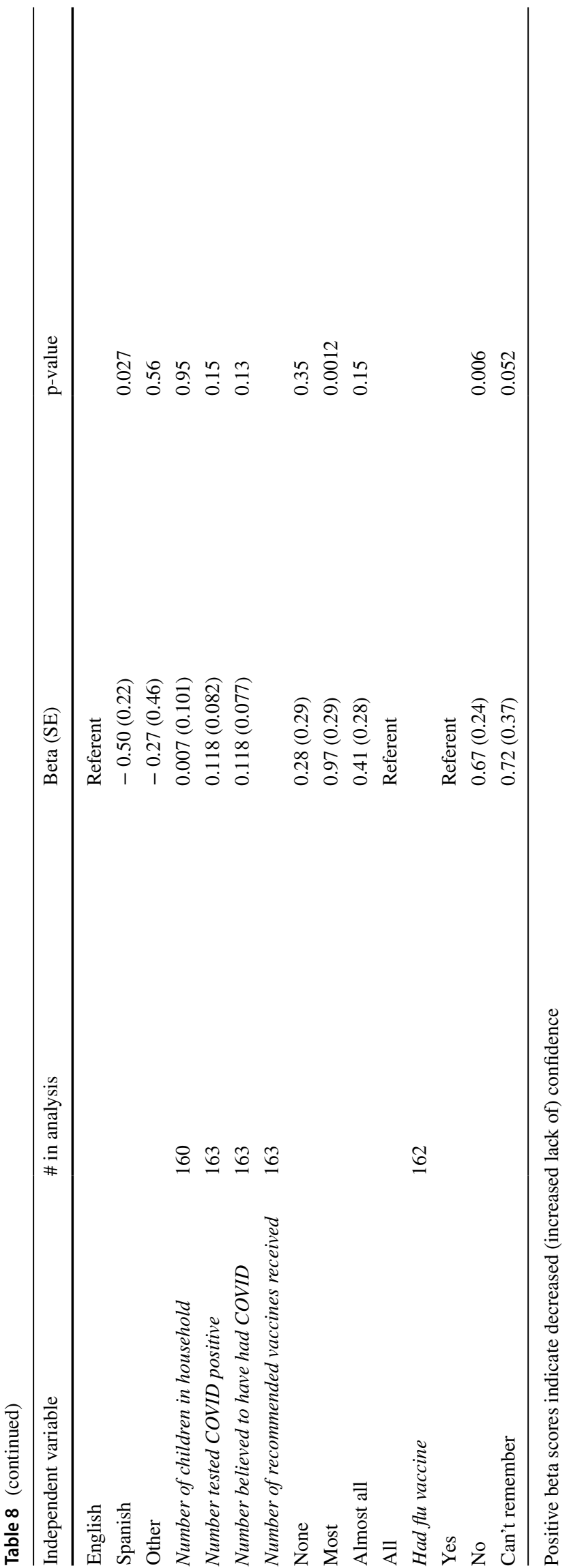

be more vaccine hesitant, in our study, political affiliation did not closely correlate with vaccine hesitancy.

Our study has several limitations, the major being that it is subject to recall bias regarding comparisons of vaccine hesitancy before and during the pandemic. In our analyses of intent to vaccinate before the pandemic, we were unable to correlate recalled parental reports with actual vaccination data given that it was an anonymous survey. It was challenging to classify the recalled number of vaccines received prior to the pandemic in a useful way. Our classification of a child having received "None," "Most," "Almost All," or "All" vaccines, and whether they planned to receive "None," "Most," "Almost All," or "All" vaccines may have been more meaningful if the categories were dichotomized to "None" and "More than None." For some comparative questions before and during the pandemic, there were lower response rates resulting in smaller sample size. In several circumstances, parents were unable to complete the survey once their child was called out of the waiting room. Future iterations of the survey may need to be shortened, with some questions rearranged to better prioritize certain responses.

The study is a single center study with a dominantly Hispanic population. While perspectives within this study are reflective of our patient population, they may not be entirely generalizable beyond our surrounding community. Due to limits imposed by the pandemic including closed clinic times and limited patient access, we were unable to obtain access to additional sites. Within our convenience sample, we had an uneven distribution of patients from general pediatrics (inpatient and outpatient) and subspecialty clinics. In the future, it may be beneficial to conduct subgroup analyses of whether these settings differed in childhood vaccine hesitancy. Finally, the time span of the survey was September 2020 and February 2021, which spanned before and after the release of the COVID-19 vaccines to the general public. Future studies may involve time-dependent analyses of how the availability of the COVID-19 vaccine may have affected parental perspectives on childhood vaccine hesitancy.

\section{Conclusion}

Childhood vaccine hesitancy is a topic of crucial importance, especially during the COVID-19 pandemic. During the pandemic, overall vaccine hesitancy and risk perception have increased, although vaccine confidence and intent to vaccinate remains unchanged. Vaccine hesitancy appears to be driven by risk perception regarding vaccine safety profiles. Despite misgivings, vaccine hesitancy does not necessarily translate into decreased intent to vaccinate, and parents continue to assign great importance to routine childhood vaccines and influenza vaccines. Vaccine hesitancy is significantly associated with different household income 


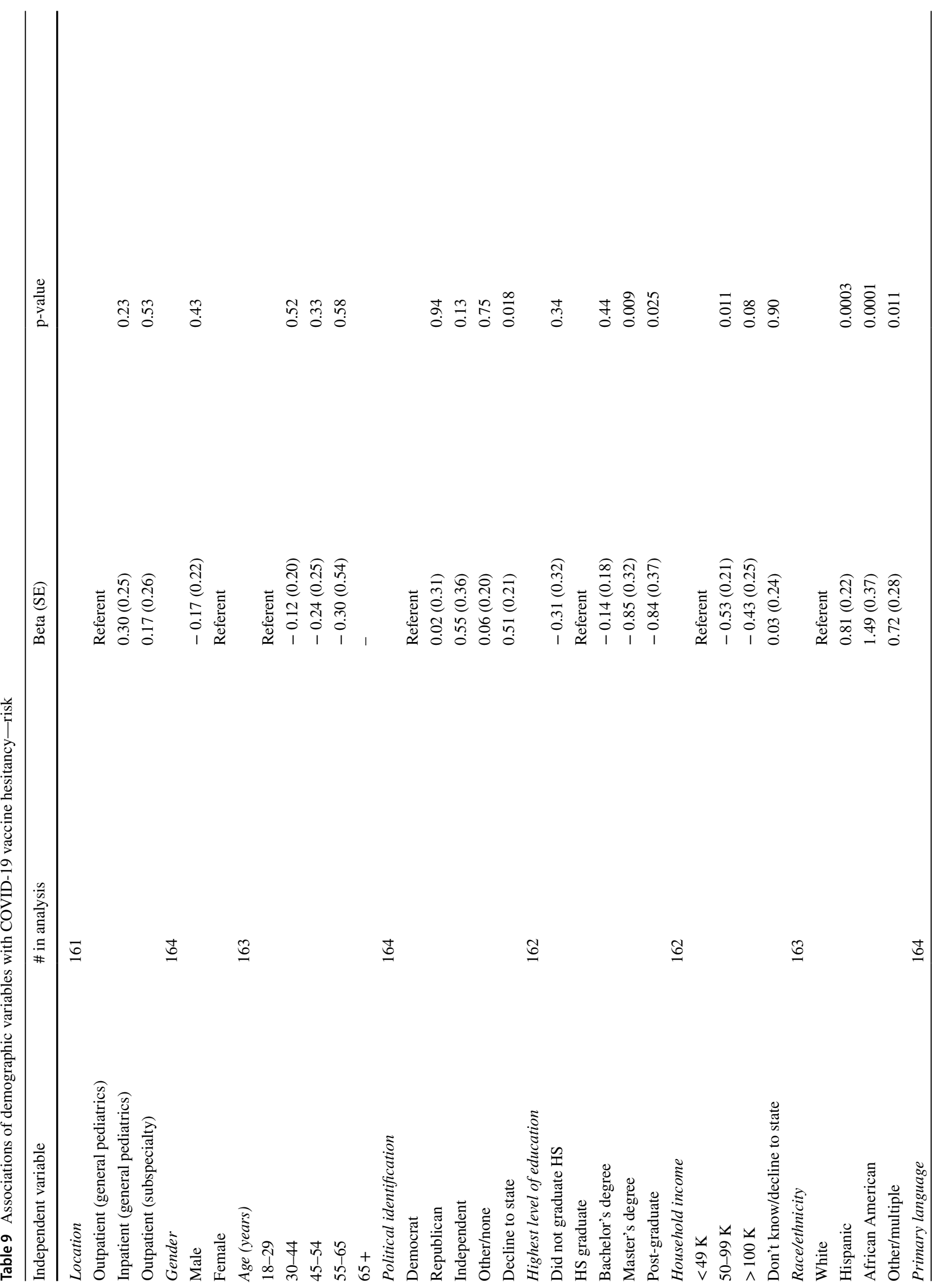




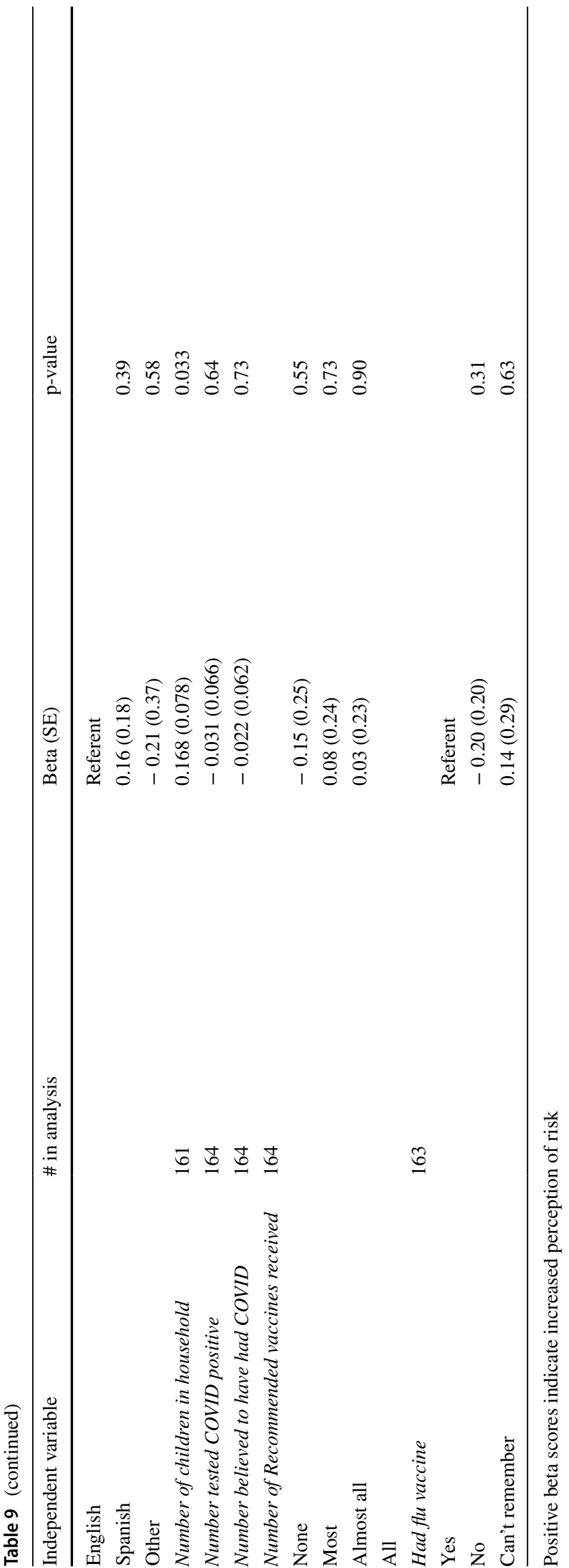

levels and race, suggesting that different strategies for different demographic groups may be needed to target effective communication about vaccines. With this study, we hope to establish a future framework to maintain pediatric immunizations and improve population health outcomes.

Acknowledgements The authors wish to thank Dr. Jeffrey Bender, Dr. Sindhu Mohandas, Dr. Michelle Kipke, and Dr. Jennifer Unger for their input in survey design. We would like to express our gratitude to Jean Meadows, Alina Fajardo, and Carmen Salas for their help in coordinating Medical Spanish translations.

Author Contributions Dr. Neely, Dr. Anand, Dr. He, and Dr. Mack conceptualized and designed the project. Dr. He and Dr. Lewis obtained survey data. Dr. Mack performed statistical analyses of the data. Dr. He drafted the initial manuscript, which underwent review by all co-authors.

Funding This work was supported by Southern California Clinical and Translational Science Institute Voucher Program [Grant No. 8092000-000013161-1020] and by Grants UL1TR001855 and UL1TR000130 from the National Center for Advancing Translational Science (NCATS) of the U.S. National Institutes of Health. The content is solely the responsibility of the authors and does not necessarily represent the official views of the National Institutes of Health.

Data Availability Not applicable.

Code Availability Not applicable.

\section{Declarations}

Conflict of interest All authors declare that they have no commercial or other associations that might pose a conflict of interests.

Ethical Approval Not applicable.

Consent to Participate Not applicable.

Consent for Publication Not applicable.

\section{References}

1. Santoli, J. M., Lindley, M. C., DeSilva, M. B., Kharbanda, E. O., Daley, M. F., Galloway, L., et al. (2020). Effects of the COVID-19 pandemic on routine pediatric vaccine ordering and administration-United States, 2020. MMWR, 69(19), 591-593.

2. Bramer, C. A., Kimmins, L. M., Swanson, R., Kuo, J., Vranesich, P., Jacques-Carroll, L. A., et al. (2020). Decline in child vaccination coverage during the COVID-19 pandemic-Michigan Care Improvement Registry, May 2016-May 2020. MMWR, 69(20), 630-631.

3. MacDonald, N. E., Eskola, J., Liang, X., Chaudhuri, M., Dube, E., Gellin, B., et al. (2015). Vaccine hesitancy: Definition, scope and determinants. Vaccine, 33(34), 4161-4164.

4. Kempe, A., Saville, A. W., Albertin, C., Zimet, G., Breck, A., Helmkamp, L., et al. (2020). Parental hesitancy about routine childhood and influenza vaccinations: A national survey [Internet]. Pediatrics, 146, e20193852. 
5. The Lancet Child \& Adolescent Health. (2019). Vaccine hesitancy: A generation at risk. Lancet Child \& Adolescent Health [Internet]., 3(5), 281. https://doi.org/10.1016/S2352-4642(19) 30092-6

6. Helmkamp, L. J., Szilagyi, P. G., Zimet, G., Saville, A. W., Gurfinkel, D., Albertin, C., et al. (2021). A validated modification of the vaccine hesitancy scale for childhood, influenza and HPV vaccines. Vaccine [Internet], 39(13), 1831-1839. https://doi.org/10. 1016/j.vaccine.2021.02.039

7. Santibanez, T. A., Nguyen, K. H., Greby, S. M., Fisher, A., Scanlon, P., Bhatt, A., et al. (2020). Parental vaccine hesitancy and childhood influenza vaccination. Pediatrics, https://doi.org/10. 1542/peds.2020-007609

8. Yigit, M., Ozkaya-Parlakay, A., \& Senel, E. (2021). Evaluation of COVID-19 vaccine refusal in parents. The Pediatric Infectious Disease Journal, 40(4), e134-e136.

9. Rhodes, M. E., Sundstrom, B., Ritter, E., McKeever, B. W., \& McKeever, R. (2020). Preparing for A COVID-19 vaccine: A mixed methods study of vaccine hesitant parents. Journal of Health Communication, 25(10), 831-837.

10. Goldman, R. D., McGregor, S., Marneni, S. R., Katsuta, T., Griffiths, M. A., Hall, J. E., et al. (2021). Willingness to vaccinate children against influenza after the coronavirus disease 2019 pandemic. Journal of Pediatrics, 228, 87-93.e2.

11. Larson, H. J., Jarrett, C., Schulz, W. S., Chaudhuri, M., Zhou, Y., Dube, E., et al. (2015). Measuring vaccine hesitancy: The development of a survey tool. Vaccine, 33(34), 4165-4175.

12. Shapiro, G. K., Tatar, O., Dube, E., Amsel, R., Knauper, B., Naz, A., et al. (2018). The vaccine hesitancy scale: Psychometric properties and validation. Vaccine, 36(5), 660-667.
13. Helmkamp, L. J., Szilagyi, P. G., Zimet, G., Saville, A. W., Gurfinkel, D., Albertin, C., et al. (2021). A validated modification of the vaccine hesitancy scale for childhood, influenza and HPV vaccines. Vaccine, 39(13), 1831-1839.

14. Kuppalli, K., Brett-Major, D. M., \& Smith, T. C. (2021). COVID19 vaccine acceptance: We need to start now. Open Forum Infectious Diseases, 8, 2-4.

15. Wheelock, A., \& Ives, J. (2021). Vaccine confidence, public understanding and probity: Time for a shift in focus? Journal of Medical Ethics [Internet]. 1-6. http://www.ncbi.nlm.nih.gov/ pubmed/33687913

16. Germani, F., \& Biller-Andorno, N. (2021). The anti-vaccination infodemic on social media: A behavioral analysis. PLOS ONE, 16, 02474642.

17. Pullan, S., \& Dey, M. (2021). Vaccine hesitancy and anti-vaccination in the time of COVID-19: A Google Trends analysis. Vaccine, 10, 20. https://doi.org/10.1016/j.vaccine.2021.03.019

18. Boyle, J., Berman, L., Nowak, G. J., Iachan, R., Middleton, D., \& Deng, Y. (2020). An assessment of parents' childhood immunization beliefs, intentions, and behaviors using a smartphone panel. Vaccine [Internet], 38(10), 2416-23. https://doi.org/10.1016/j. vaccine.2020.01.032

19. Khubchandani, J., Sharma, S., Price, J. H., Wiblishauser, M. J., Sharma, M., \& Webb, F. J. (2021). COVID-19 vaccination hesitancy in the United States: A rapid national assessment. Journal of Community Health [Internet]., 46(2), 270-7. https://doi.org/10. 1007/s10900-020-00958-x

Publisher's Note Springer Nature remains neutral with regard to jurisdictional claims in published maps and institutional affiliations. 